\title{
Generation of pulsar radio emission
}

\author{
Yu.E. Lyubarskii \\ Institute of Radio Astronomy, Krasnoznamyonnaya St.4, 310002, \\ Kharkov, Ukraine
}

\begin{abstract}
The generation of radio emission from plasma waves excited by two-stream instability in pulsar magnetospheres is considered. Induced scattering transforms the excited longitudinal waves into waves that escape freely in the form of transverse electromagnetic waves. It is shown that the spectrum and the luminosity of the generated radio emission are compatible with those observed.
\end{abstract}

Strong coherent radio emissions from pulsars are believed to result from the development of plasma instabilities in the magnetosphere of the neutron star. The two-stream instability, which readily excites strong plasma oscillations, is among the most widely discussed. Conditions for the development of the twostream instability in pulsar magnetospheres are not trivial. Possible scenarios are discussed by Usov (1987), Ursov \& Usov (1988), Lyubarskii (1992a,b, 1993), Lyubarskii \& Petrova (2000). Let us assume that the instability does develop and consider the formation of pulsar radio emission from the excited plasma waves.

The instability excites longitudinal waves in Cherenkov resonance with generating particles, $\omega=k v$. Such waves can not escape from plasma; however if the energy density of these waves is high enough, the induced scattering becomes an effective process. It is well known (see, e.g., Tsytovich 1970) that the induced scattering redistributes the wave energy towards large wavelengths (in the plasma rest frame). Inspection of the dispersion curves of the waves in the magnetospheric plasma shows that the waves eventually become superluminous. These superluminous waves escape freely in the form of vacuum transverse waves if they propagate in the curved magnetic field in plasma with density decreasing outwards. So observed radio emission may be generated as a result of induced scattering of longitudinal waves excited by the two-stream instability.

The induced scattering rate in the ultrarelativistic plasma embedded in the superstrong magnetic field may be roughly estimated as (for a more detailed analysis of the kinetic equation see Lyubarskii (1996))

$$
\frac{d W}{d t} \sim \frac{\omega_{0} W}{\gamma^{3} n m c^{2}} W
$$

where $W$ is the wave energy density, $n$ plasma number density, $\gamma$ the plasma Lorentz factor, $m$ the electron mass, $\omega_{0}=\omega_{p} \sqrt{\gamma}$ the Lorentz-shifted plasma frequency. 
The condition that the induced scattering rate is of the order of the escape rate, $c / r$, implies an estimate for the wave energy density

$$
\frac{\omega_{0} W}{\gamma^{3} n m c^{2}} \sim \frac{c}{r}
$$

Then the radio luminosity may be estimated as

$$
L \sim c W S \sim \frac{c \gamma^{2}}{\omega_{0} r} \gamma n m c^{3} S,
$$

where $S$ is the cross section of the open flux tube. Taking into account that the observed frequency $\omega=2 \pi c / \lambda$ is of the order of $\omega_{0}$, one can write

$$
L \sim \frac{\lambda \gamma^{2}}{r} L_{0}
$$

where $L_{0}=S \gamma n m c^{3}$ is the total power of the plasma flow. For the typical parameters $r \sim 10^{7}-10^{8} \mathrm{~cm}, \lambda \sim 1 \mathrm{~m}, \gamma \sim 100$ one can see that a significant fraction of the plasma energy may be converted into radio emission. Taking plasma number densities from theories of plasma generation (see, e.q., Arons 1983 ), one can obtain the radio luminosity of the order of $10^{26}-10^{29} \mathrm{erg} / \mathrm{s}$.

Taking into account the radius-to-frequency mapping, $\omega_{0} \propto r^{-3 / 2}$, one can estimate the spectrum of the outgoing radiation as

$$
\frac{d L}{d \nu}=\frac{d L}{d r} / \frac{d \nu}{d r} \propto \nu^{-4 / 3}
$$

So within the scope of the presented simple model one can obtain reasonable luminosity and spectrum of pulsar radio emission. Because the frequency of the emitted waves is close to the local plasma frequency, refraction is quite important. Detailed analysis of the wave escape, with account of refraction, polarization transfer etc., shows that morphology of the pulses, pulse width versus frequency curves, both and polarization characteristics of pulsar radio emission may be naturally exlained (Petrova \& Lyubarskii 1999).

\section{References}

Arons, J. 1983, in: Electron-Positron Pairs in Astrophysics. Eds. M.L.Burns, A.K.Harding, R.Ramaty. American Institute of Physics. P.163.

Lyubarskii, Yu.E. 1992a, A\&A, 261, 544

Lyubarskii, Yu.E. 1992b, A\&A, 265, L33

Lyubarskii, Yu.E. 1993, Astron.Lett., 19, 14

Lyubarskii, Yu.E. 1996, A\&A, 308, 809

Lyubarskii, Yu.E. \& Petrova, S.A. 2000, A\&A, in press

Petrova, S.A. \& Lyubarskii, Yu.E. 1999, this volume

Tsytovich, V.N. 1970, Non-linear Effects in Plasma. Plenum Press

Ursov, V.V. \& Usov, V.V. 1988, Ap\&SS, 140, 325

Usov, V.V. 1987, ApJ, 320, 333 